\title{
A Study on User Interface Design According to 3D Menu Type and Control Type for 3D Displays
}

\author{
Jaeho Kim ${ }^{1}$, Sungwook Jung ${ }^{1}$, Jaeho Choe ${ }^{2}$, Eui S. Jung ${ }^{3}$ \\ ${ }^{1}$ Graduate School of Industrial Management and Security, Korea University, Seoul, 136-701 \\ ${ }^{2}$ Department of Industrial and system Engineering, Daejin University, Pocheon, 487-711 \\ ${ }^{3}$ Division of Information Management Engineering, Korea University, Seoul, 136-701
}

\begin{abstract}
Objective: We proposed an effective 3D menu manipulation alternative with a usability test. This was performed in a 3D environment with 3D menu and control manipulation methods. Background: As 3D stereoscopic displays became generalized, various 3D applications were being used not just movies, games, but also mobile contents and advertisements. However, when a user interface was designed in a 3D environment, it was lacked that a clear standard and result of an efficiency and usability as like a 2D environment. Method: We implemented 9 kinds of 3D menu types based on 3D menu hierarchies, menu layouts and dimensions. And we extracted 3 representative control types in a 3D environment. We performed usability evaluation with full factorial design for 27 menu alternatives with 2 types of menu manipulation tasks. In this condition, we measured the performance time, subjective discomfort and eye-fatigue. Results: A control type had the significant effect, and the effect of menu types had the different result depending on given tasks. Conclusion: This experiment showed the interaction of menu and control types depended on 3D menu manipulation tasks were significant in a 3D user interface design. Therefore, as a purpose of a 3D menu manipulation task, 3D menu types and control types were should be considered. Application: We supposed this result was should be applied in a 3D menu contents design.
\end{abstract}

Keywords: 3D Menu, 3D Controller, User Interface Design, Usability Evaluation

\section{Introduction}

최근 $3 \mathrm{D} \mathrm{TV}$, 모니터 등 양안식 $3 \mathrm{D}$ 디스플레이 $(3 \mathrm{D}$ Stereoscopic Display)의 보급으로 전방 표시 장치(Headup Display)나 3D Cave와 같은 복잡한 장비 없이도 $3 \mathrm{D}$ 안경과 디스플레이를 통해 $3 \mathrm{D}$ 컨텐츠의 경험이 확대되고 있으며, $3 \mathrm{D}$ 영화, 게임, 광고 등 상용화된 $3 \mathrm{D}$ 어플리케이션 들이 출시되고 있다. 양안식 $3 \mathrm{D}$ 디스플레이의 경우 가상의 3 차원 공간에서 가상의 물체(Virtual Object)를 조작하고 시야의 방향을 전환하여 볼 수 있으며 목표물을 직접 가리 킬 수도 있는 등 $2 \mathrm{D}$ 환경보다 현실감을 증가시켜 3 차원
공간의 지각 능력에 대한 이해를 향상시킬 수 있다는 장점이 있다(Ware and Franck, 1996).

이러한 $3 \mathrm{D}$ 컨텐츠를 효율적으로 제어하기 위한 방법 이나, 건축물, 제품 등의 설계를 $3 \mathrm{D}$ 가상 환경 (Virtual Environment)를 통해 효율적으로 수행하는 방법 등에 대 해서도 많은 연구들이 이루어 지고 있으며(Ye 등, 2007), 이처럼 다양한 $3 \mathrm{D}$ User Interface가 등장함에 따라 새로운 컨트롤이나 인터렉션 방식에 대한 연구들이 지속적으로 수 행되고 있다(Bowman etc, 2006).

그러나 이러한 연구들은 양안식 $3 \mathrm{D}$ 디스플레이 환경에서 보여지는 인터페이스의 표현방식 (Graphical User Interface) 과 컨트롤을 함께 고려하기 보다는 가상의 물체를 효과적으 
로 회전(Brookshire etc, 1992)하거나 선택(Vanaken etc, 2008 ) 또는 이동(Bowman etc, 2001) 하는 새로운 인터렉 션(컨트롤) 방식에 대한 연구가 주를 이루고 있다. 따라서 이러한 인터렉션 기술이나 방식이 실제 $3 \mathrm{D}$ 메뉴와 같은 사 용자 인터페이스와 함께 적용되기 위해서는 컨트롤방식과 다양한 인터페이스의 표현방식을 함께 고려한 사용성에 대한 연구가 필요하다. $2 \mathrm{D}$ 인터페이스 환경에서 사용되는 Pull-down이나 Pop-up 방식 등은 $3 \mathrm{D}$ 환경에서도 그대 로 적용되어질 수는 있지만, 마우스나 키보드가 $3 \mathrm{D}$ 환경에 서 유용한 방식은 아닐 수 있기 때문이다(Jacoby and Ellis, 1992).

$3 \mathrm{D}$ Computer graphics 분야에서는 $3 \mathrm{D}$ 환경에서 효율 적인 메뉴방식에 대한 연구들이 진행되어 왔다. Dachselt 와 Hübner (2007)는 [Figure 1] 과 같이 3D 커서를 이용 하여 메뉴를 선택할 수 있게 한 공간 메뉴(Floating menu) (Bowman and Wingrave, 2002), 기존의 2D 기반의 순환 메뉴방식을 확장하여 새로운 컨트롤과 접목한 스핀 메뉴 (Spin Menu) (Gerber and Bechmann, 2004) 등 1990년대 부터 개발된 $3 \mathrm{D}$ 메뉴를 정리하여 발표하였다. 또한 최근에 는 Microsoft사가 개발한 동작인식시스템인 키넥트를 이 용하여 다양한 인터페이스를 효과적으로 컨트롤하려는 노력 도 진행되고 있다.

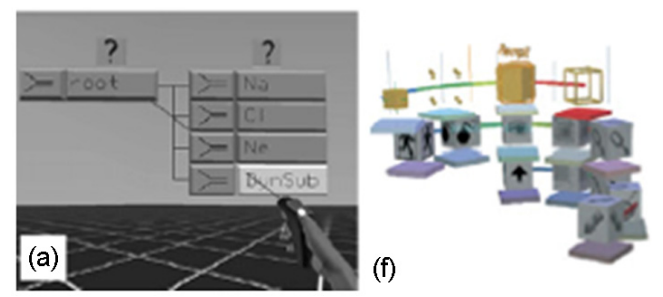

Figure 1. Floating and Spin menu (Dachselt and Hübner, 2007)

그러나 2D 환경의 User Interface Design과 비교하여 범용적인 $3 \mathrm{D}$ 메뉴 구조나 방식에 적합한 대안이나 가이드 라인에 대한 연구는 미흡한 수준이며, $\mathrm{PC}$ 의 마우스나 키보 드, $\mathrm{TV}$ 의 리모콘처럼 $\mathrm{3D}$ 환경에서 보편적으로 적합한 콘 트롤방식에 대한 연구도 아직까지 미흡한 실정이다.

본 연구에서는 $3 \mathrm{D}$ 컨텐츠나 어플리케이션에 범용적으로 적용 가능한 메뉴방식과 컨트롤에 대한 연구를 수행하였다. 먼저 $2 \mathrm{D}$ 환경에서 널리 사용되고 있는 메뉴의 방식과 선 택방법을 분류하고, $3 \mathrm{D}$ 환경에서 이를 표현하기 위한 $\mathrm{GUI}$ 방식을 기존의 연구 결과를 바탕으로 추출하였다. 또한, $3 \mathrm{D}$ $\mathrm{GUI}$ 를 제어하기 위하여 개발된 다양한 컨트롤방식을 조사 하고 그 중에서 범용적으로 사용 가능한 대안을 선정하였다.
다음으로, $3 \mathrm{D}$ 환경에서 일반적으로 사용될 수 있는 메뉴 대 안을 구성하고 선정된 컨트롤들을 사용하여 수행도 및 불편 도 평가 실험을 수행하였다. 이를 통하여 $2 \mathrm{D}$ 환경의 메뉴 방식을 $3 \mathrm{D}$ 환경에서 구현하였을 경우 적합한 컨트롤방식을 알아보았으며, 결과를 바탕으로 $3 \mathrm{D}$ 환경에서 $\mathrm{GUI}$ 와 컨트롤 의 인터렉션에 영향을 미치는 디자인 변수에 대한 분석을 실시하였다.

\section{Methods}

\subsection{D Menu taxonomy}

2D에서 가장 많이 사용되고 있는 메뉴방식으로는 Pulldown, Pop-up 및 Stack 방식(또는 탭방식) 메뉴가 있다. [Figure 2] 과 같이 Pull-down 방식은 하위 메뉴가 상위 메뉴의 하단에 주로 고정되어 나타나는 형태이며, Pop-up 방식은 하위 메뉴가 커서가 있는 곳에서 활성화 되거나 화면 의 중앙에서 활성화 되는 형태이다(Kim 등, 2000). Stack 방식은 Pop-up 방식의 확장 형태로 여러 개의 Pop-up 메뉴를 쉽게 탐색할 수 있다는 장점이 있는 반면, 너무 많은 메뉴창이 화면상에 표시되어 사용자에게 혼란을 야기시킬 수 있다는 단점이 있다. 최근에는 [Figure 2]의 오른쪽과 같 이 메뉴를 3 차원 공간에서 보이듯이 표현하여 이전의 메뉴를 쉽게 활성화할 수 있는 방식의 Stack 메뉴도 개발되었다.

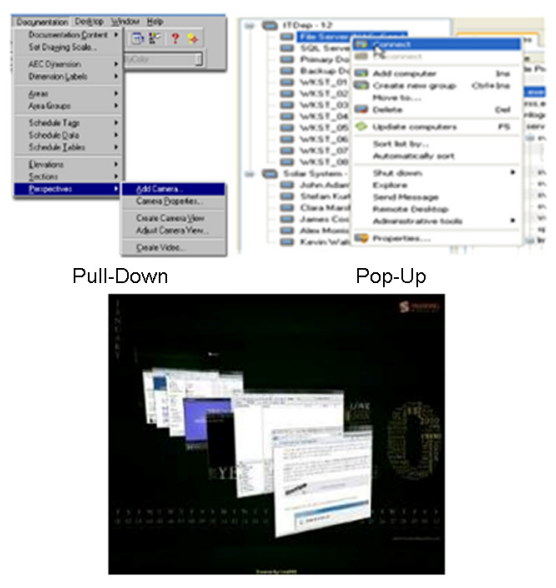

Figure 2. 2D menu representation type

$3 \mathrm{D}$ 환경에서 구현한 효율적인 메뉴방식 중 하나는 메뉴 아이템들을 직선이 아닌 원형방식으로 보여주고 회전 조작 을 통하여 선택하는 방법이다. Gerber와 Becnmann(2005) 은 [Figure 3]에서와 같이 $3 \mathrm{D}$ 환경에서 효율적인 계층적 
스핀 메뉴방식을 구현하고, 3가지의 메뉴 구조(교차방식, 집 중방식, Stack 방식)에 대한 수행도를 측정하는 연구를 수행 하였다. 연구 결과 메뉴의 아이템을 원형으로 배열하는 집중 방식과 이전에 선택한 메뉴로 쉽게 돌아갈 수 있는 Stack 방식의 수행도가 우수한 것으로 분석되었다.
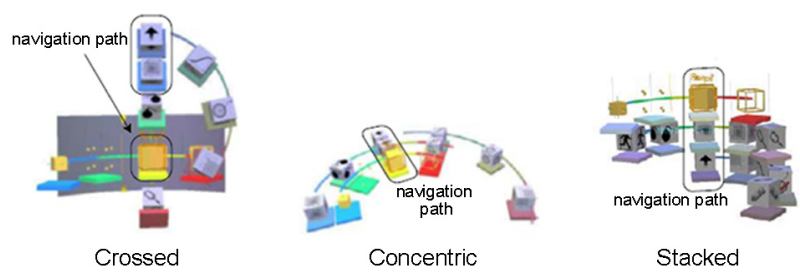

Figure 3. 3D Hierarchical spin menu (Gerber and Bechmann, 2005)

$3 \mathrm{D}$ 환경에서 메뉴의 특성을 결정하는 중요한 요소로 차 원성(Dimensionality)이 있다. Mine(1995)은 선택 가능한 모든 아이템이 하나의 평면상에서 보여지는 것을 1 차원 메 뉴로, 아이템을 선택하기 위하여 다음 메뉴를 다른 평면상에 서 불러오는 것을 2 차원 메뉴로 정의하였다. 2 차원 메뉴의 대표적인 방식으로는 Block 방식, Cluster 방식, TreeCube 방식이 있다(A Leal etc, 2009).

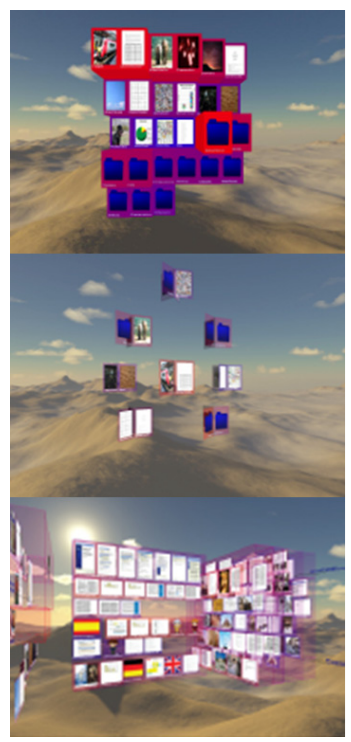

Figure 4. Block, Cluster and TreeCube menu

\subsection{D Menu design alternatives}

본 연구에서는 기존의 연구 결과들을 토대로 일반적인 상 황에서 사용 가능한 $3 \mathrm{D}$ 메뉴를 분류하기 위한 기준으로 다
음과 같은 3 가지를 선정하였다. 메뉴의 계층구조의 경우 $2 \mathrm{D}$ 환경에서 많이 사용하는 방식을 사용하였고, 메뉴 레이아웃 은, Gerber와 Becnmann(2005)의 연구 결과를 토대로 선 형과 원형방식을 선정하였다. 메뉴 차원성은 Mine(1995)의 연구를 토대로 1 차원과 2차원으로 구성하였다.

- 메뉴계층구조: 하위 메뉴의 활성화 형태에 따른 분류 기 준으로 Pull-down 방식, Pop-up 방식과 Stack 방식으 로 분류

- 메뉴 레이아웃: 상위 메뉴가 Display에 표현되어지는 배 열의 형상으로서 선형방식과 원형방식으로 분류

- 메뉴 차원성: $3 \mathrm{D}$ 메뉴 구조에서 표현 가능한 특징으로서 1 차원 메뉴와 2차원 메뉴로 분류

위의 3 가지 $3 \mathrm{D}$ 메뉴 분류 기준으로 메뉴계층구조 3 가지, 메뉴 레이아웃 방식 2 가지, 메뉴 차원성 2 가지의 총 12 가 지 조합 중 [Table 1] 과 같이 9가지 조건을 실험 대안으로 선정하였으며, 메뉴 차원성과 메뉴 레이아웃 방식의 조합을 메뉴방식이라고 정의하였다. 2 차원 원형 대안의 경우는 1 차 원 원형 대안과 특징이 크게 다르지 않아 제외하였다.

Table 1. Menu alternatives

\begin{tabular}{c|c|c}
\hline \multicolumn{2}{c|}{ Menu type } & Menu hierarchy \\
\hline \multirow{3}{*}{$1 \mathrm{D}$} & \multirow{3}{*}{ Linear } & Pull-down \\
\cline { 3 - 3 } & & Pop-up \\
\cline { 3 - 3 } & & Stack \\
\cline { 3 - 3 } $2 \mathrm{D}$ & & Pull-down \\
\cline { 3 - 3 } & & Pop-up \\
\cline { 3 - 3 } & & Stack \\
\hline \multirow{2}{*}{$1 \mathrm{D}$} & \multirow{3}{*}{ Rotary } & Pull-down \\
\cline { 3 - 3 } & & Pop-up \\
\hline
\end{tabular}

2 차원 메뉴의 경우 기존 $3 \mathrm{D}$ 컨텐츠에서 많이 사용되는 TreeCube 방식으로 대안을 구현하였다. 선형 Stack 방식 의 경우 이전에 선택한 메뉴들이 현재 활성화되어 있는 메 뉴의 뒷 공간으로 물러가도록 디자인 하였으며, 원형 Stack 의 경우는 뒷 공간에 다른 메뉴들이 있기 때문에 화면 좌측 으로 이동하도록 하였다.

[Figure 5] 은 1차원_선형 방식, 2차원 선형 방식과 1차원 -원형 방식에 대한 각각의 3 가지 메뉴계층구조 대안과 구현 사례이다. 

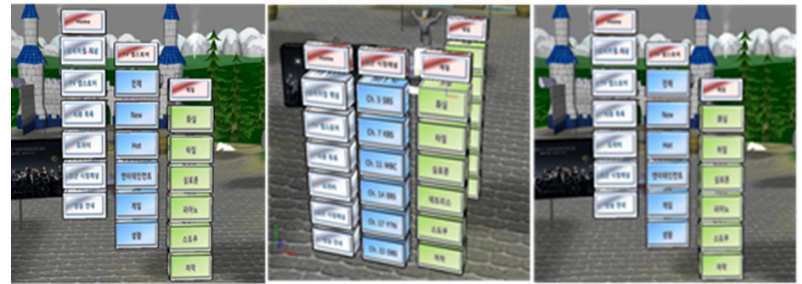

1차원_선형Pull-down

1차원_선형_Stack

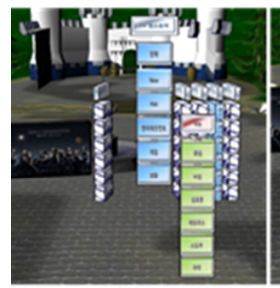

2차원_Linear_Pull-down

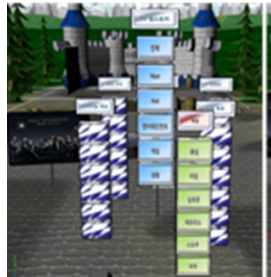

1차원_Rotary_Pull-down

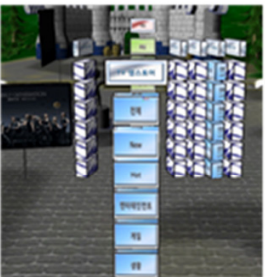

2차원_Linear_Stack

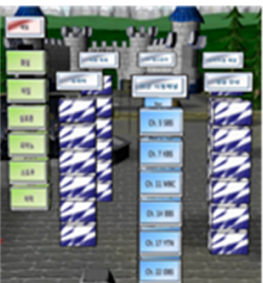

1차원_Rotary_Stack
1차원_선형Pull-down

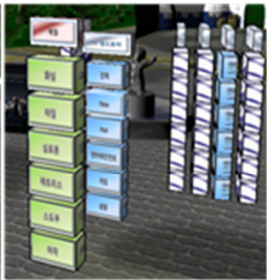

2차원_Linear_Pop-up

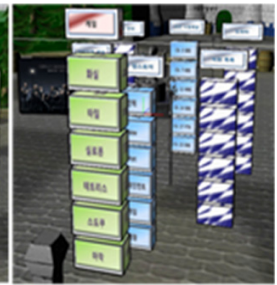

1차원_Rotary_Pop-up

Figure 5. 9 alternatives of 3D menus

\subsection{D Control type alternatives}

$3 \mathrm{D}$ 환경에서 메뉴 선택 작업을 위하여 개발된 초기 기 술로 레이저 건을 이용한 Ray casting technique이 있다 (Liang and Green, 1994). 사용자는 총 모양의 컨트롤로 3D Display 상의 물체를 포인팅하여 선택할 수 있는 것으로 2차원 환경에서 마우스와 포인터 개념의 확장 형태라고 할 수 있다. 이는 Object가 작거나 다른 Object에 가려져 있 을 경우 조작이 힘들다는 단점이 있다(Bowman 등, 2001).

현재 많이 연구되고 있는 3D 컨트롤방식은 3D cursor를 사용자 손의 움직임을 통하여 제어하는 기술이다(Poupyrev, 1996). Go-go technique이라고 불리는 이 기술은 현재까 지 정확도를 높이려는 노력이 지속적으로 진행되고 있다.

마지막으로 마우스나 키보드를 확장해서 $3 \mathrm{D}$ 에 적용하려 는 기술로 Logitech사의 3D 마우스나 Nintendo사의 WII 리모트 컨트롤러와 같은 $2 \mathrm{D}$ 컨트롤방식에 키를 추가하여 $3 \mathrm{D}$ 환경을 제어할 수 있는 기술들이 있다.

위의 3 가지 컨트롤방식은 일반적인 $3 \mathrm{D} \mathrm{GUI}$ 환경에 적 용 가능할 뿐 아니라 현재도 지속적으로 연구되는 컨트롤 방식으로 향후에도 $3 \mathrm{D}$ Display와 함께 사용될 가능성이 크다고 판단된다. 따라서 본 연구에서는 Ray-casting 방식,

Hand-motion 방식, Depth 방향(3D 상의 앞, 뒤 방향) 컨 트롤을 추가한 Keypad 방식을 실험 대안으로 선정하였다.

Ray-casting 방식의 컨트롤은 Istation-Wing이라는 기 기를 변경하여 제작하였다. 무선방식으로 상하좌우 4 방향으 로 컨트롤을 움직여 포인트를 이동시키고 타겟에 맞추어 버 튼을 누르면 메뉴가 선택되도록 하였다.

Hand-motion 방식의 컨트롤은 Microsoft의 키넥트 기 기를 이용하여 실험을 실시하였다. 키넥트는 센서를 통해 사 용자의 동작을 인식해 타겟을 움직이는 방식으로, 본 실험에 서는 양손의 움직임으로 과제를 수행하도록 하였다. 오른손 좌우 움직임을 최상위 메뉴 간의 이동으로, 오른손 상하 움 직임을 하위 메뉴의 이동으로 설정하였으며, 왼손을 앞으로 밀면 타겟 활성화를, 몸 쪽으로 당기면 메뉴를 불러올 수 있 도록 하였다.

Keypad 방식의 컨트롤은 방향키의 좌우를 메뉴 간 이동 으로, 상하를 메뉴 내의 이동으로 설정하였다. 메뉴 활성화 는 방아쇠로 조작하고 별도의 버튼으로 메뉴를 불러올 수 있도록 하였다.

\subsection{Experimental fesign}

실험은 [Table 1]에서와 같이 3수준의 메뉴방식(1차 원 선형, 2 차원 선형, 1 차원 원형), 3 수준의 메뉴계층구조 (Pull-down, Pop-up, Stack)와 3수준의 컨트롤방식 (Ray-casting, Hand-motion, Keypad)의 3개 독립변수 의 Full Factorial Design으로 설계하였다. 총 27개 실험 조건에 대해 Within Subject Design으로 실험을 수행하였 으며, 학습 효과를 최소화하기 위하여 Randomized Block Design으로 실시하였다.

실험 과제는 [Figure 6] 와 같이 최상위 메뉴에서 최하 위 메뉴까지 이동하여 목표 아이템을 선택하는 Simple Navigation Task과, Simple Navigation Task를 수행한 후 다시 최상위 메뉴 중 다른 아이템을 선택하여 2nd 레벨의 메뉴까지 이동하고 다시 이전에 선택한 메뉴 중에서 최하위 메뉴까지 찾아가는 Complex Navigation Task의 2가지 과 제를 수행하였다. Complex Navigation Task의 경우 사용자

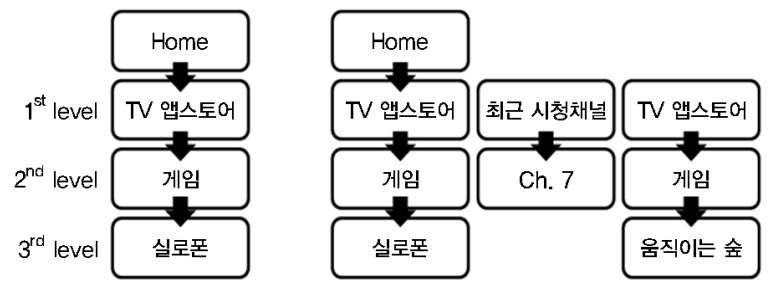

Figure 6. Tasks of 3D menu manipulations 
가 2D 환경에서 일반적으로 수행하는 Multi-tasking 환경 을 고려하기 위하여 디자인하였다.

종속변수로서 정량적인 평가 기준인 과제 수행시간을 측 정하였으며, 정성적인 평가 기준으로 불편도와 눈의 피로도 를 9점 스케일 리커트 척도를 사용하여 평가하였다.

\subsection{Subjects and experimental environments}

$3 \mathrm{D}$ 디스플레이 환경을 한 번 이상 접해본 20 30대 총 27 명의 피실험자를 대상으로 실험을 수행하였으며, 참여한 피실험자의 평균 연령은 28.4세였다.

실험은 [Figure 7] 과 같은 환경의 Virtual Reality Room 에서 듀얼 프로젝터를 스크린에 투사하고, 편광방식의 글 래스 타입을 이용하였다. 3D 구현 및 구동 프로그램은 Dassault Systems사의 3DVIA Virtools를 사용하였다. 피 실험자는 Display와 $4 \mathrm{~m}$ 떨어진 거리에 앉아서 실험을 수행 하였다.

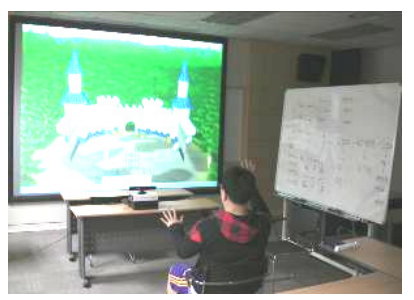

Figure 7. Experimental environments

\section{Results}

\subsection{Simple navigation task}

Simple Navigation Task의 수행시간에 대한 분산분석 결과는 [Table 2] 와 같다. 유의수준 0.05 수준에서 메뉴방 식과 컨트롤방식의 주 효과와 2 차와 3 차 모든 교호작용이 유의한 것으로 나타났다. $F$ 값을 기준으로 각 변수가 수행 시간에 미치는 영향력은 컨트롤방식 $(F=534.08)>$ 메뉴방 식 $(F=4.18)$ 의 순으로 나타났다.

컨트롤방식의 경우 [Figure 8] 과 같이 Ray-casting 방식 (7.00초) < Keypad 방식 (13.46초) < Hand-motion 방식 (17.38초) 순으로 작업수행시간이 나타났으며, LSD 기법 을 이용한 사후분석결과 세 가지 방식 간에 모두 유의한 차 이가 있는 것으로 분석되었다.

메뉴방식에 따른 수행시간의 차이는 [Figure 9] 과 같이 2차원_선형의 수행시간이 가장 작게 나타났으며, 사후분석
결과 2차원 선형과 다른 두 방식간에 유의한 차이를 보였다.

Table 2. ANOVA results of simple navigation task

*: Significant at $\alpha=0.05$

\begin{tabular}{l|c|r|c}
\hline \multicolumn{1}{c|}{ Source } & DF & \multicolumn{1}{c|}{$\mathrm{F}$} & $p$-vaule \\
\hline Menu type & 2 & 4.18 & $0.02^{*}$ \\
\hline Menu hierarchy & 2 & 2.72 & 0.75 \\
\hline Control type & 2 & 534.08 & $0.00^{*}$ \\
\hline Menu type * Menu hierarchy & 4 & 67.30 & $0.00^{*}$ \\
\hline Menu type * Control type & 4 & 4.57 & $0.00^{*}$ \\
\hline Menu hierarchy * Control type & 4 & 2.39 & $0.05^{*}$ \\
$\begin{array}{l}\text { Menu type * Menu hierarchy } \\
\text { * Control type }\end{array}$ & 8 & 6.57 & $0.00^{*}$ \\
\hline
\end{tabular}

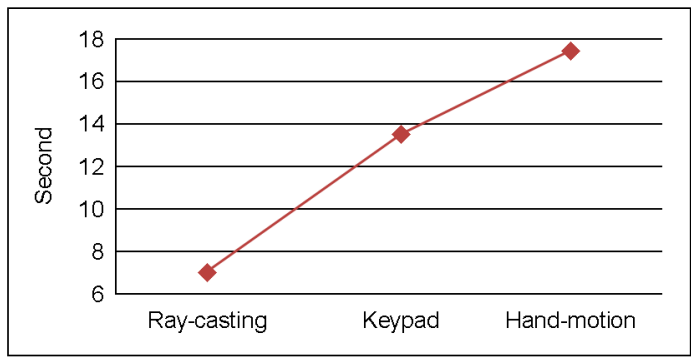

Figure 8. Performance time of control types

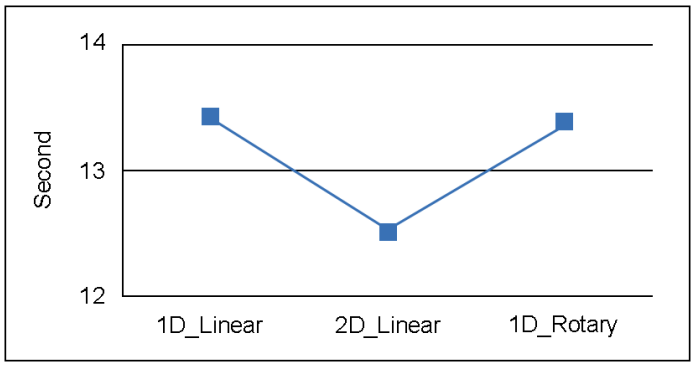

Figure 9. Performance time of menu types

메뉴방식과 메뉴계층구조 간의 교호작용의 경우 [Figure 10 ] 와 같이 Pop-up과 Stack 방식은 2차원_선형 < 1 차원 -원형 < 1 차원_선형의 순으로 작업수행시간의 변화가 같은 경향을 나타냈으나, Pull-down 방식은 1차원_선형 < 2 차원 _선형 < 1차원_원형의 순으로 다른 경향을 보였다.

메뉴방식과 컨트롤방식의 교호작용의 경우 [Figure 11] 과 같이 Ray-casting 방식은 메뉴방식에 상관없이 유사한 작업수행시간을 보였으며, Hand-motion 컨트롤방식이 수 행시간이 가장 길었으며 메뉴방식 중에는 2 차원_선형이 가 
장 짧은 수행시간으로 나타났다.

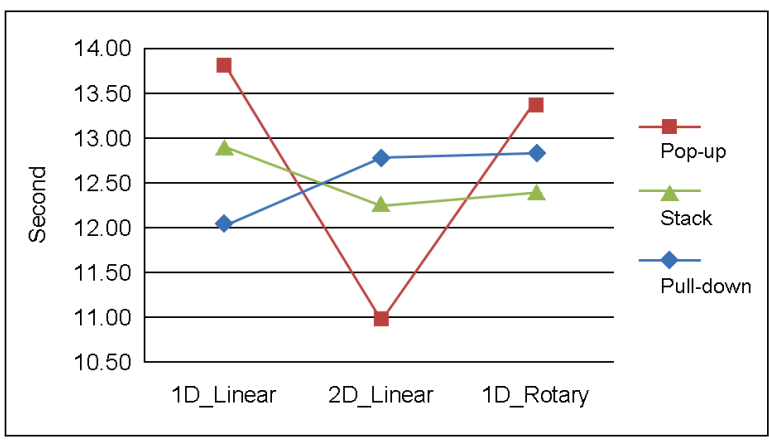

Figure 10. Performance time of the interaction (a menu type and menu hierarchy)

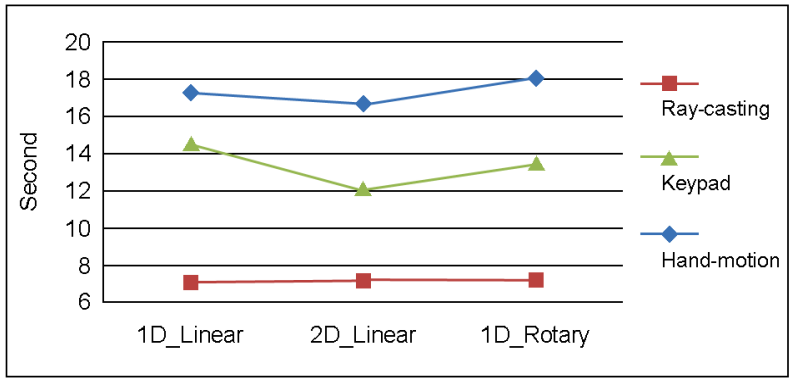

Figure 11. Performance time of the interaction (menu and control type)

메뉴방식, 컨트롤방식, 메뉴계층구조 간의 3 차 교호작용은 [Table 3] 과 같이 1차원_선형 방식의 Pull-down 방식에 Ray-casting 방식의 컨트롤을 사용한 경우가 6.22초로 가 장 적은 작업수행시간을 보였다. 그러나 1 위와 2 위 간의 통 계적 차이를 나타나지 않았으며 3 위, 4 위와 5 위도 통계적으 로 유의한 차이를 보이지 않는 것으로 분석되었다.

Table 3. Performance time of simple navigation task

$(\mathrm{sec})$

\begin{tabular}{c|c|c|c|c}
\hline & Menu type & $\begin{array}{c}\text { Menu } \\
\text { hierarchy }\end{array}$ & Control type & Time \\
\hline 1 & 1D_Linear & Pull-down & Ray-casting & 6.22 \\
\hline 2 & 1D_Linear & Stack & Ray-casting & 6.58 \\
\hline 3 & 2D_Linear & Pull-down & Ray-casting & 6.81 \\
\hline 4 & 2D_Linear & Stack & Ray-casting & 6.85 \\
\hline 5 & 1D_Linear & Pop-up & Ray-casting & 6.99 \\
\hline
\end{tabular}

또한 [Figure 12] 과 같이 메뉴방식과 컨트롤방식의 교호
작용은 2차원_선형 $\mathrm{Pop}-\mathrm{up}$ 이 가장 우수한 것으로 나타났 지만 Keypad, Ray-casting의 경우 Stack, Pull-down 방 식에 대한 작업수행시간에 통계적으로 유의한 차이가 없었 다. 단지, Hand-motion의 경우 Pop-up 방식이 다른 방식 보다 작업수행시간이 빠른 것으로 나타났다.

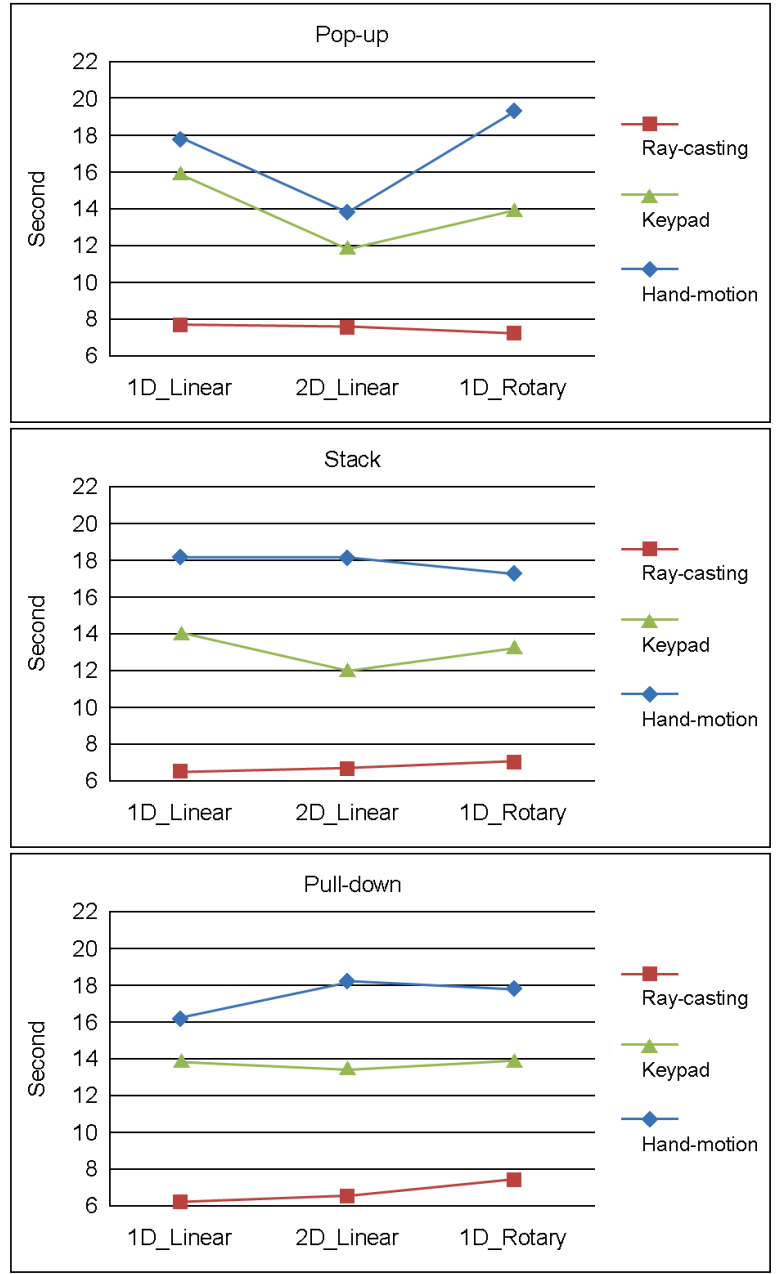

Figure 12. Performance time of the interaction (menu type, control type and menu hierarchy)

\subsection{Complex navigation task}

Complex Navigation Task 수행시간에 대한 분산분석 결과는 [Table 4] 와 같다. 유의수준 0.05 기준으로 주 효과 는 메뉴계층구조와 컨트롤방식이 유의한 것으로 나타났으며, 2 차 교호작용과 3 차 교호작용 모두 유의하게 분석되었다.

각 변수의 영향력은 컨트롤방식 $(F=173.62)>$ 메뉴계층 구조 $(F=39.99)$ 의 순으로 나타났다.

Simple Navigation Task의 경우는 메뉴방식과 컨트롤방 
식이 유의하게 나타난 반면 Complex Navigation Task에 서는 메뉴계층구조와 컨트롤방식이 유의하게 나타났다.

Table 4. ANOVA results of complex navigation task

*: Significant at $\alpha=0.05$

\begin{tabular}{l|c|c|c}
\hline Source & DF & F & $p$-vaule \\
\hline Menu type & 2 & 1.56 & 0.22 \\
\hline Menu hierarchy & 2 & 102.88 & $0.00^{*}$ \\
\hline Control type & 2 & 654.52 & $0.00^{*}$ \\
\hline Menu type * Menu hierarchy & 4 & 23.68 & $0.00^{*}$ \\
\hline Menu type * Control type & 4 & 19.16 & $0.00^{*}$ \\
\hline Menu hierarchy * Control type & 4 & 13.76 & $0.00^{*}$ \\
\hline $\begin{array}{l}\text { Menu type * Menu hierarchy } \\
\text { * Control type }\end{array}$ & 8 & 4.09 & $0.00^{*}$ \\
\hline
\end{tabular}

컨트롤방식의 경우는 [Figure 13] 와 같이 Simple Navigation Task와 같은 경향으로 Ray-casting 방식(21.22초) <Keypad 방식 (35.88초) < Hand-motion 방식(47.28초) 순으로 작업수행시간이 나타났다.

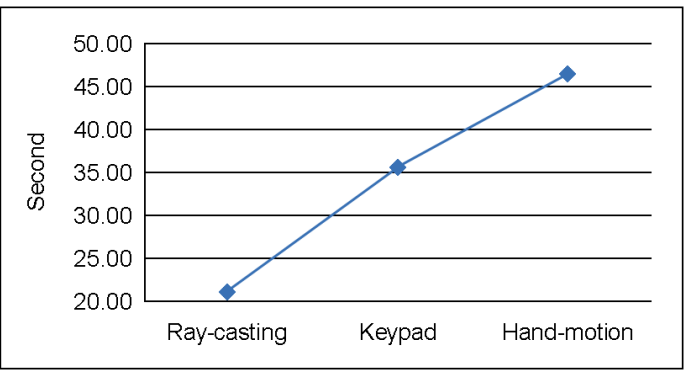

Figure 13. Performance time of control types

메뉴계층구조의 경우 [Figure 14] 과 같이 Stack 방식 (30.11초) < Pop-Up 방식 (38.04초) < Pull-Down 방식 (36.22초) 순으로 작업수행시간이 나타났다. 사후분석결과

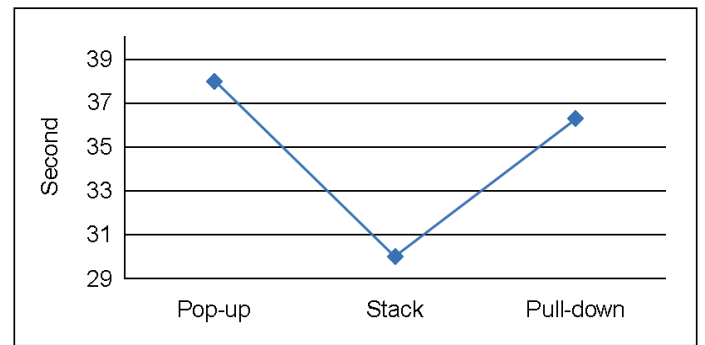

Figure 14. Performance time of menu hierarchies
세 가지 방식 모두 유의한 차이가 있는 것으로 분석되었다. 메뉴방식과 메뉴계층구조의 교호작용 분석결과 [Figure 15 ] 와 같이 $\mathrm{Pop}-\mathrm{up}$ 방식의 경우는 2차원_선형 < 1 차원 원형 < 1 차원_선형의 순으로 작업수행시간이 적었고, Stack 방식의 경우 1 차원 원형과 1 차원 선형 간에는 작업수행시간 의 통계적으로 유의한 차이를 보이지 않았고 2차원_선형이 가장 긴 작업수행시간이 나타났다. Pull-down 방식의 경우 $\mathrm{Pop}-\mathrm{up}$ 방식과는 반대의 유형이 나타나 1 차원_선형 < 1 차 원_원형 < 2 차원_선형의 순으로 작업수행시간이 나타났다.

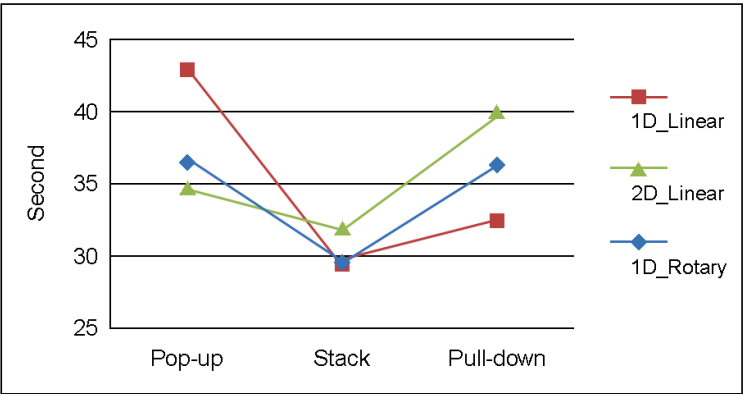

Figure 15. Performance time of the interaction (menu type and menu hierarchy)

메뉴방식과 컨트롤방식의 교호작용 분석결과 [Figure 16] 와 같이 메뉴방식의 영향에 상관없이 컨트롤방식의 종류에 따라 작업수행시간이 달라지는 것으로 나타났다.

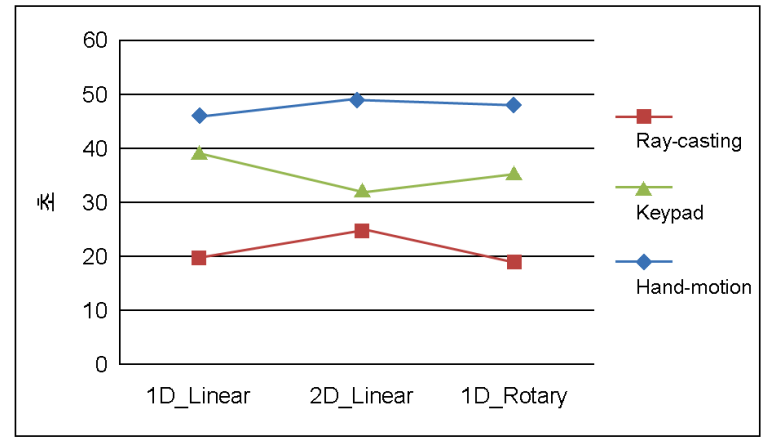

Figure 16. Performance time of the interaction (menu and control type)

메뉴계층구조와 컨트롤방식의 교호작용 분석결과 [Figure 17] 과 같이 모든 컨트롤방식에서 Stack < Pull-down < Pop-up의 순으로 작업수행시간이 나타났다.

메뉴방식, 메뉴계층구조와 컨트롤방식간의 교호작용 분석 결과 [Table 5] 와 같이 1차원_선형 방식의 Stack 방식을 Ray-casting 방식의 컨트롤을 사용한 실험이 17.11초로 
가장 적은 작업수행시간을 보였으며, 사후분석결과 1 3위는 유의한 차이를 보이지 않았고 4,5 위 간에도 유의한 차이를 보이지 않았다.

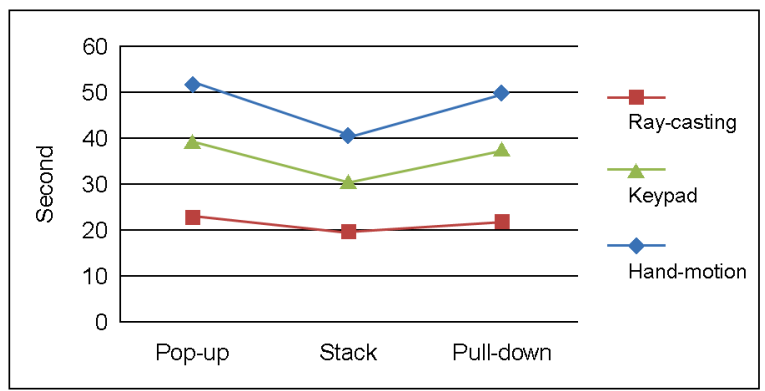

Figure 17. Performance time of the interaction between a control type and menu hierarchy

Table 5. Performance time of complex navigation task

(sec)

\begin{tabular}{c|c|c|c|c}
\hline 순위 & 메뉴방식 & $\begin{array}{c}\text { 메뉴계층 } \\
\text { 구조 }\end{array}$ & 컨트롤 방식 & $\begin{array}{c}\text { 작업수행 } \\
\text { 시간 }\end{array}$ \\
\hline 1 & 1D_Linear & Stack & Ray-casting & 17.11 \\
\hline 2 & 1D_Rotary & Stack & Ray-casting & 17.69 \\
\hline 3 & 1D_Linear & Pull-down & Ray-casting & 18.12 \\
\hline 4 & 1D_Rotary & Pop-up & Ray-casting & 19.42 \\
\hline 5 & 1D_Rotary & Pull-down & Ray-casting & 20.11 \\
\hline
\end{tabular}

[Figure 18] 과 같이 Stack과 Pull-down의 경우는 컨 트롤방식이 메뉴방식에 대하여 작업수행시간이 통계적 차 이를 나타내지 못했지만 Pop-up의 경우 1차원_선형에서 Keypad 방식과 Hand-motion 방식이 다른 두 메뉴방식에 비하여 긴 작업수행시간을 나타냈다.

\subsection{Discomfort for menu manipulations}

불편도에 대한 분산분석 결과 [Table 6] 과 같이 유의수준 0.05 기준으로 3 가지 주 효과와 메뉴방식과 메뉴계층구조, 메뉴방식과 컨트롤방식 간의 2 차 교호작용이 유의한 차이를 보였다.

각 변수 간의 영향력은 컨트롤방식 $(F=44.92)>$ 메뉴방 식 $(F=11.40)>$ 메뉴계층구조 $(F=4.65)$ 의 순으로 나타 났다

위의 두 Task와 비교하면 불편도의 경우는 메뉴방식과 메뉴계층구조가 모두 유의한 차이를 보인 반면에 메뉴방식, 메뉴계층구조와 컨트롤간의 3 차 교호작용은 유의한 차이를 보이지 않았다.

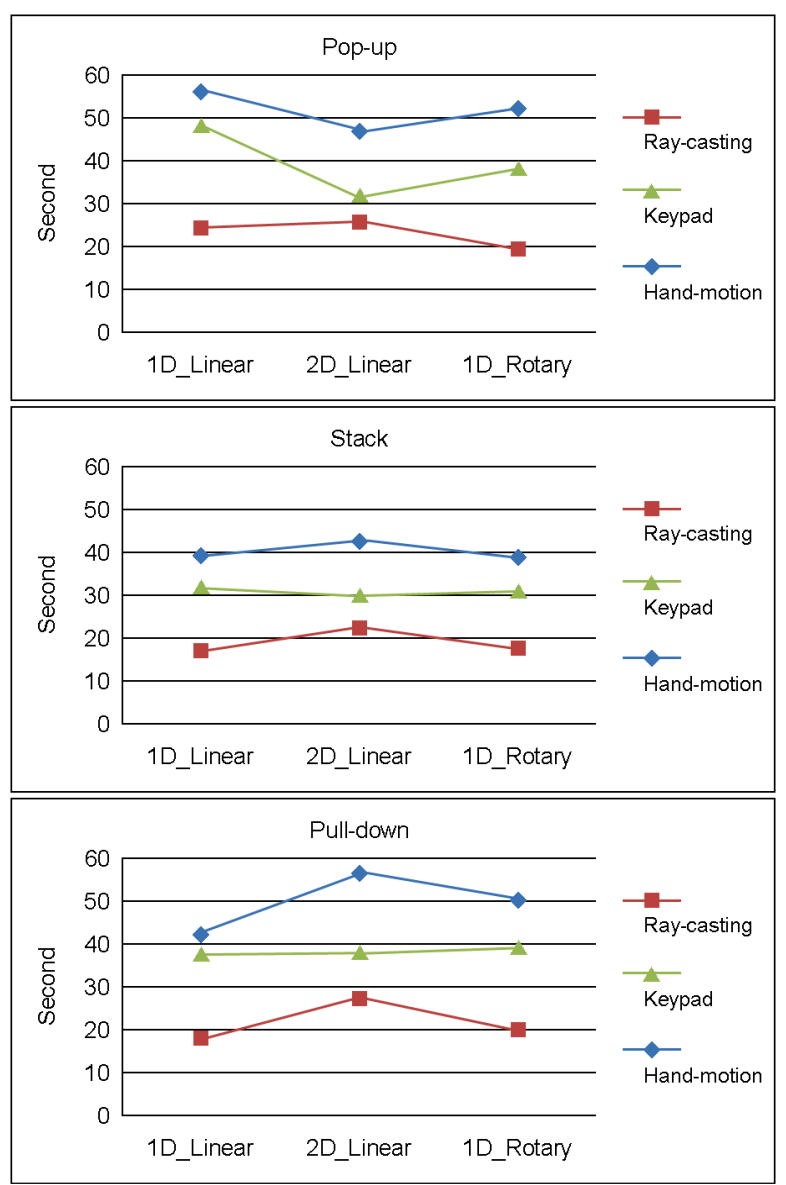

Figure 18. Performance time of the interaction (menu type, control type and menu hierarchy)

Table 6. ANOVA results of menu manipulations

\begin{tabular}{l|c|c|c}
\hline \multicolumn{3}{|c}{ *: Significant at $\alpha=0.05$} \\
\hline Source & DF & F & $p$-vaule \\
\hline Menu type & 2 & 11.40 & $0.00^{*}$ \\
\hline Menu hierarchy & 2 & 4.65 & $0.01^{*}$ \\
\hline Control type & 2 & 44.92 & $0.00^{*}$ \\
\hline Menu type * Menu hierarchy & 4 & 15.04 & $0.00^{*}$ \\
\hline Menu type * Control type & 4 & 3.34 & $0.01 *$ \\
\hline Menu hierarchy * Control type & 4 & 0.24 & 0.91 \\
\hline $\begin{array}{l}\text { Menu type * Menu hierarchy } \\
\text { * Control type }\end{array}$ & 8 & 1.37 & 0.21 \\
\hline
\end{tabular}

컨트롤방식에 따른 불편도의 차이는 [Figure 19] 와 같이 수행시간 결과와 같은 경향을 나타내었다.

메뉴방식의 경우 [Figure 20] 과 같이 2차원 선형 방식이 4.74점으로 가장 불편한 것으로 분석되었으며, 사후분석결 
과 다른 두 방식에 대하여 유의한 차이를 보였다.

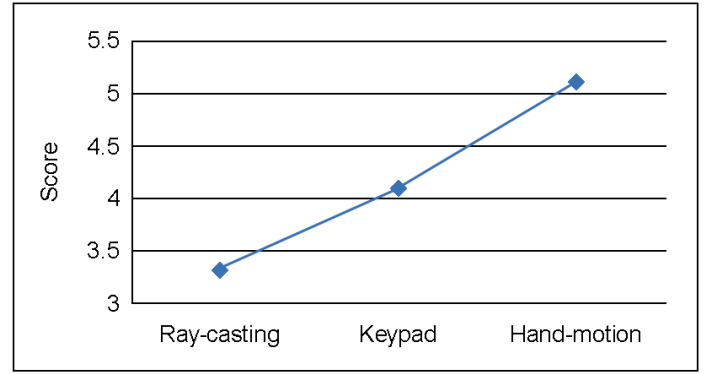

Figure 19. The means of control types

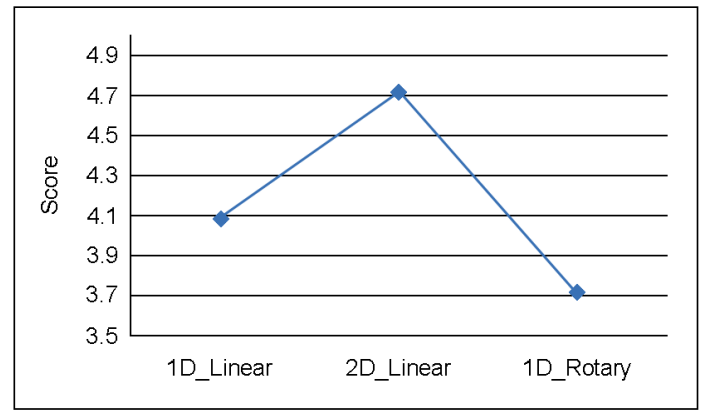

Figure 20. Results of menu type

메뉴계층구조의 경우 [Figure 21] 과 같이 Pop-up 방식 이 가장 불편하며, 사후분석결과 다른 두 방식에 대하여 유 의한 차이를 보였다.

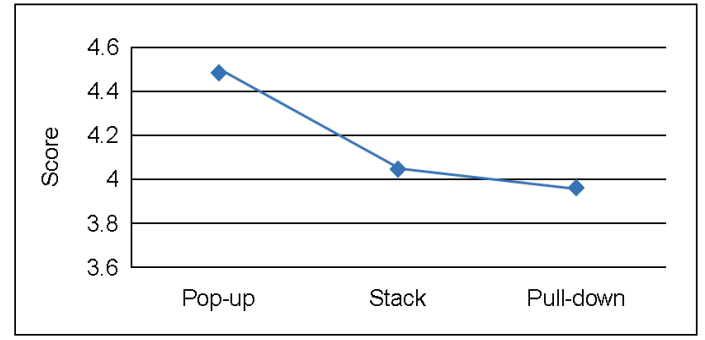

Figure 21. The means of menu hierarchies

메뉴방식과 메뉴계층구조의 교호작용 분석결과 [Figure 22] 과 같이 Pop-up 방식의 경우 1차원_선형 방식> 2차원 _선형 방식 > 1차원_원형 방식의 순으로 나타났고, Stack 방식의 경우 2 차원_선형 방식 > 1 차원_원형 방식 > 1 차원 선형 방식 순으로 불편도의 가장 높은 것으로 분석되었다. Pull-down 방식에서는 1차원_선형 방식과 1차원_원형 방
식에는 유의한 차이를 보이지 않았고 2 차원 선형이 가장 불 편한 것으로 나타났다.

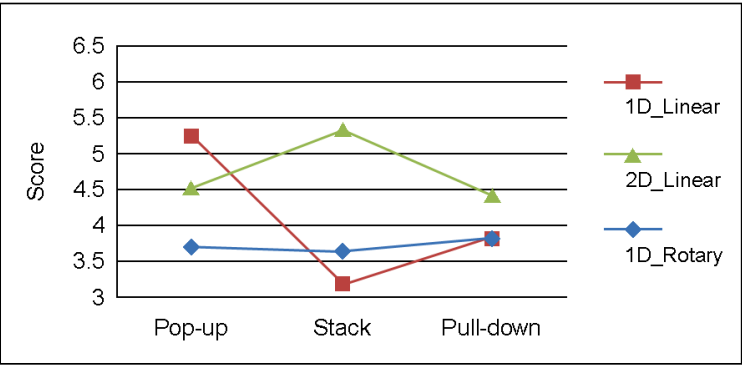

Figure 22. The means of the interaction (menu type and menu hierarchy)

메뉴방식과 컨트롤방식의 교호작용 분석결과 [Figure 23] 와 같이 2차원 선형방식에서 Ray-casting 방식이 Keypad 방식보다 불편도가 높게 나와 다른 메뉴방식의 경향과 다르 게 나타났다.

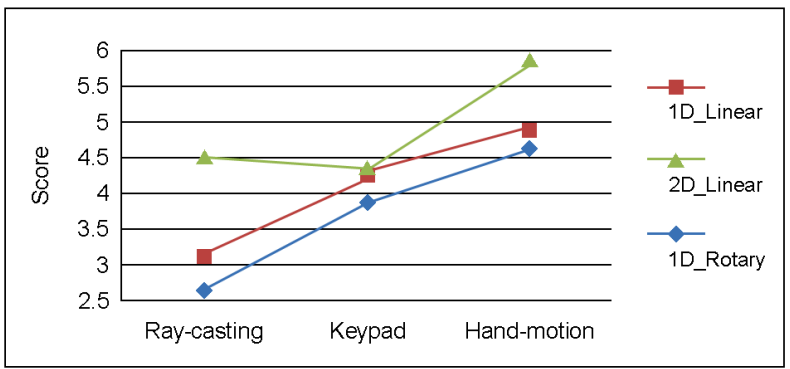

Figure 23. The means of the interaction (a menu and control type)

\subsection{Eye-Fatigue for Menu Manipulations}

눈 피로도에 대한 분산분석 결과 [Table 7] 과 같이 유의 수준 0.1 기준으로 메뉴방식과 메뉴계층구조의 주 효과만이 유의한 것으로 나타났으며 교호작용은 유의한 차이를 보이

Table 7. ANOVA results of eye-fatigue for menu manipulations

\begin{tabular}{l|c|c|c}
\multicolumn{4}{r}{$\begin{array}{r}\text { *: Significant at } \alpha=0.05 \\
\text { **: Significant at } \alpha=0.10\end{array}$} \\
\hline Source & DF & F & $p$-vaule \\
\hline Menu type & 2 & 2.76 & $0.07^{* *}$ \\
\hline Menu hierarchy & 2 & 10.21 & $0.00^{*}$ \\
\hline Menu type * Menu hierarchy & 4 & 1.67 & 0.16
\end{tabular}


지 않았다. 각 변수간의 영향력은 메뉴계층구조 $(F=10.21)$

$>$ 메뉴방식 $(F=2.76)$ 의 순으로 나타났다.

메뉴방식의 경우 [Figure 24] 과 같이 1차원_선형 방식이 가장 낮은 점수를 보였으며, 사후분석결과 다른 두 방식에 대하여 유의한 차이를 보였다.

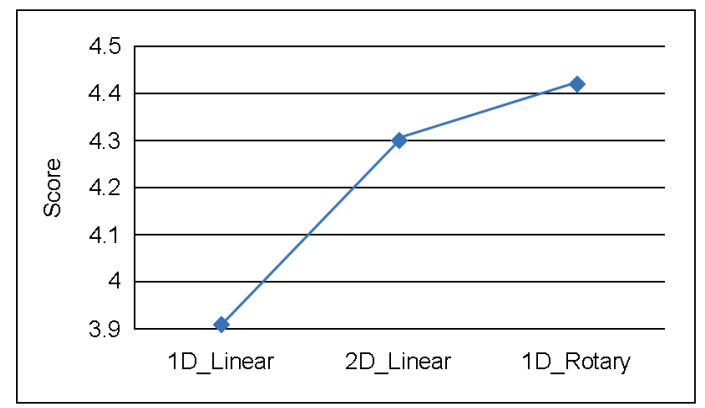

Figure 24. Results of menu type

메뉴계층구조의 경우 [Figure 25] 과 같이 Pop-up 방식 (4.49점) > Stack 방식 (4.06점) > Pull-down 방식 (3.98 점) 순으로 불편도가 나타났고, 사후분석결과 Pop-up 방 식만이 다른 두 방식에 대하여 유의한 차이를 보였다.

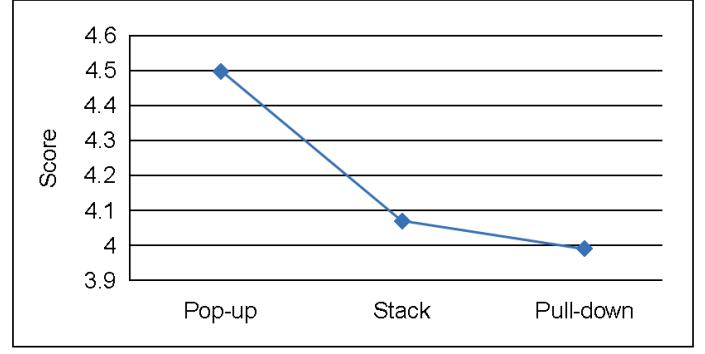

Figure 25. Results of menu hierarchy

\section{Discussions}

\subsection{Results of dependent variables}

[Table 8] 을 통하여 종속변수에 대한 결과를 보면, Task 의 수행에서는 컨트롤러방식이 가장 큰 $F$ 값을 보이는 것을 보아 가장 큰 영향력을 주는 것으로 판단된다. 메뉴계층구조 의 경우 $2 \mathrm{D}$ 환경에서 일반적으로 수행하는 Multi-tasking 과 백트렉킹에 의하여 영향력이 달라지는 것으로 판단된다. Multi-tasking과 백트렉킹 수행이 없는 Simple Navigation
Task의 경우는 실험결과 메뉴계층구조가 유의하지 않는 것 으로 나타났지만, Complex Navigation Task의 경우는 메 뉴계층구조에서 높은 $\mathrm{F}$ 값을 나타내는 것을 볼 수 있었다.

사용성 불편도의 경우 메뉴방식의 $F$ 값이 메뉴계층구조 보다 높게 나타난 것으로 보아 메뉴의 차원성에 영향을 받 는 것으로 판단할 수 있다. 앞의 결과에서 나타나듯 차원성 이 높은 2차원_선형방식이 가장 높은 불편도를 나타낸다.

눈의 피로도는 사용성 불편도의 경향과는 반대로 메뉴의 차원성 보다는 메뉴계층구조에 영향을 받는 것으로 판단할 수 있다.

Table 8. Significant difference between dependent variables

\begin{tabular}{|c|c|c|c|c|}
\hline & $\begin{array}{l}\text { Simple } \\
\text { navigation } \\
\text { task } \\
\text { (F) }\end{array}$ & $\begin{array}{l}\text { Complex } \\
\text { navigation } \\
\text { task } \\
\text { (F) }\end{array}$ & $\begin{array}{l}\text { Discomfort } \\
\text { for menu } \\
\text { manipulations } \\
\text { (F) }\end{array}$ & $\begin{array}{l}\text { Eye- } \\
\text { fatigue } \\
\text { (F) }\end{array}$ \\
\hline Menu type & $V_{\text {(4.18) }}$ & & $V_{(11.4)}$ & $V_{(2.76)}$ \\
\hline Menu hierarchy & & $V(102.88)$ & $V(4.65)$ & $\boldsymbol{V}(10.21)$ \\
\hline Control type & $\nu(534.08)$ & $\boldsymbol{V}(654.52)$ & $\nu(44.92)$ & \\
\hline $\begin{array}{l}\text { Menu type } \\
* \text { Menu hierarchy }\end{array}$ & $v \quad(67.3)$ & $V_{(23.68)}$ & $V_{(15.04)}$ & \\
\hline $\begin{array}{l}\text { Menu type } \\
* \text { Control type }\end{array}$ & $(4.57)$ & $V(19.16)$ & $V(3.34)$ & \\
\hline $\begin{array}{l}\text { Menu hierarchy } \\
* \text { Control type }\end{array}$ & $V \quad(2.39)$ & $V(13 / 76)$ & & \\
\hline $\begin{array}{l}\text { Menu type } \\
* \text { Menu hierarchy } \\
* \text { Control type }\end{array}$ & $V \quad(6.57)$ & $\sqrt{(4 / 09)}$ & & \\
\hline
\end{tabular}

[Table 9]를 보면 모든 종속변수에서 Ray-casting 방 식이 좋은 변수로 선정되었다. 하지만, 사용성 불편도에서 다른 대안들 보다 Multi-tasking과 백트렉킹 수행이 많 은 1차원_선형_Stack_Keypad이 낮은 불편도 점수를 받아 Multi-tasking과 백트렉킹 수행이 많은 경우에 Keypad 방 식에 대한 추후 연구가 필요할 것으로 보인다.

Simple Navigation Task의 경우는 메뉴 레이아웃 방식이 선형이 좋은 대안으로 선전되었지만, Complex Navigation Task에서는 원형의 레이아웃이 높은 순위를 차지하여 메뉴 의 레이아웃 방식이 Multi-tasking과 백트렉킹 수행에 영 향을 받는 것으로 판단된다.

눈의 피로도의 경우는 각 메뉴방식들의 Pull-down 방식 이 적은 피로도 점수를 보여 $3 \mathrm{D}$ 공간의 깊이(Depth)를 사 용하는 것이 눈의 피로도를 증가시키는 것으로 판단된다. 
Table 9. The best alternatives

\begin{tabular}{c|l|l}
\hline & \multicolumn{1}{|c|}{ Simple navigation task } & \multicolumn{1}{c}{ Complex navigation task } \\
\hline 1 & 1D_Linear_Pull-down_Ray-casting & 1D_Linear_Stack_Ray-casting \\
\hline 2 & 1D_Linear_Stack_Ray-casting & 1D_Rotary_Stack_Ray-casting \\
\hline 3 & 2D_Linear_Pull-down_Ray-casting & 1D_Linear_Pull-down_Ray-casting \\
\hline 4 & 2D_Linear_Stack_Ray-casting & 1D_Rotary_Pop-up_Ray-casting \\
\hline 5 & 1D_Rotary_Pop-up_Ray-casting & 1D_Rotary_Pull-down_Ray-casting \\
\hline & Discomfort for menu manipulations & \multicolumn{1}{|c}{ Eye-fatigue } \\
\hline 1 & 1D_Rotary_Pull-down_Ray-casting & 1D_Linear_Pull-down \\
\hline 2 & 1D_Rotary_Stack_Ray-casting & 2D_Linear_Pull-down \\
\hline 3 & 1D_Linear_Pull-down_Ray-casting & 1D_Linear_Pull-down \\
\hline 4 & 1D_Rotary_Pop-up_Ray-casting & \\
\hline 5 & 1D_Linear_Stack_Keypad & \\
\hline
\end{tabular}

\subsection{Proposals for 3D UI Design}

$3 \mathrm{D}$ 환경에서 메뉴 간의 이동과 선택의 경우 3 가지 컨트 롤방식은 Ray-casting 방식이 9가지 메뉴 대안 모두에서 좋은 것으로 나타났으며, Hand Motion 방식과 Keypad 방 식의 경우는 대안과 Task에 따른 경향의 차이가 존재하였 다. 3D 환경에서 Selection task의 경우 Ray-casting 방식 이 Go-go technique 방식보다 좋다는 Bowman, Johnson and Hodges (2001)의 연구 결과와도 일치한다.

Complex Navigation Task에서는 메뉴계층구조의 영향 력이 크게 나타났으며, Simple Navigation Task에서는 메 뉴의 메뉴방식에 대한 영향력이 나타나는 것을 알 수 있다. 이러한 결과는 예를 들어, 3D TV에서 채널 이동이나 컨텐츠 선택 등의 간단한 메뉴 구조의 경우 메뉴의 형태와 차원성에 대한 GUI 디자인 형태를 가장 중요하게 고려하여야 하며, 인터넷 검색이나 시스템 조작 등의 비교적 어려운 Task의 경우 $2 \mathrm{D}$ 환경에서와 마찬가지로 메뉴계층구조를 가장 중요 하게 고려하여야 한다는 사실을 의미한다.

반면에, 눈의 피로도의 측면에서 1차원_원형_Stack이 좋 은 사용성과 낮은 불편도를 보였지만, 1 차원 메뉴와 Pulldown 방식보다 시각적 불편도를 더 많이 느낀다는 것으로 나타났다. 따라서 $3 \mathrm{D}$ GUI를 디자인할 경우에 효율적인 메 뉴방식과 함께 시각적 피로도를 최소화할 수 있는 디자인이 중요하다고 할 수 있다. 예컨대 3D Display를 오랫동안 주 시해야 하는 Task의 경우는 1차원 메뉴와 Pull-down 방 식이 사용자들에게 더 높은 편안함을 가져다 줄 수 있을 것 이며, 게임과 같은 빠른 반응을 요구하는 메뉴방식의 경우는 2차원 메뉴의 Stack 방식이 더욱 유용할 수 있을 것이다.

$3 \mathrm{D}$ 메뉴를 설계할 때 고려해야 할 사항으로 첫째, Task 가 단순한 Task인지 복잡한 Task인지를 생각해야 할 것이
고 둘째, 사용성과 만족도를 위해 차원성을 부여할 것인지 피로도를 고려하여 $2 \mathrm{D}$ 와 같이 차원성을 부여하지 않을 것 인지에 대하여 생각해봐야 할 것이다. 마지막으로 차원성에 따른 컨트롤방식의 선택을 생각해야 할 것이다. 물론, 본 실험에서는 Ray-casting 방식이 좋은 사용성을 보였지만, Ray-casting 방식의 단점인 Object가 작거나 다른 Object 에 가려져 있을 경우 조작이 힘들다는 점이 2차원_선형의 경우 Ray-casting이 Keypad보다 불편도가 높게 나타났으 므로 차원성 부여 메뉴에서는 Keypad 방식을 고려하는 것 이 좋을 것으로 판단된다.

Select Task의 이외에 회전을 하거나 사물을 확대, 축 소하는 Task의 경우에는 Ray-casting 방식보다 Handmotion 방식이 더 좋다는 연구 결과가 있으므로(Bowman 등, 2007) Ray-casting 방식을 변경한 좀더 효율적인 컨 트롤방식이 연구되어야 할 것으로 판단된다.

본 연구의 결과가 현재 개발되거나 상용화되고 있는 모든 $3 \mathrm{D}$ UI 설계의 평가 기준이 될 수는 없을 것이다. 성공적인 $3 \mathrm{D}$ UI 설계를 위해서는 Domain과 Task에 적합한 인터렉 션 기술이나 컨트롤을 설계하는 것이 바람직하다(Bowman 등, 2006). 그러나 현재 3D UI 디자이너들이 UI를 설계할 경우 고려해야 할 컨트롤과 메뉴방식 간의 중요한 설계 특 성과 비교 기준에 대한 연구는 많이 이루어지지 못하고 있 다. 따라서 본 연구는 $2 \mathrm{D}$ 환경에서 UI 디자인 특성과 같이 $3 \mathrm{D}$ 환경에서의 UI 디자인 특성에 대한 기초적인 연구로서 $\mathrm{UI}$ 디자이너들에게 좀 더 실질적이고 적용 가능한 정보를 제공할 것으로 기대된다.

\section{References}

Anamary, L., Chadwick, A., Wingrave, J. and LaViola Jr., "Initial Explorations into the User Experience of 3D File Browsing.", Proceedings of the 23rd British HCI Group Annual Conference on People and Computers: Celebrating People and Technology, 2009.

Bowman, D. A. and Wingrave, C. A., "Design and Evaluation of Menu Systems for Immersive Virtual Environments", Proceedings of the Virtual Reality 2001 Conference (VR'01), (pp.149), 2001.

Bowman, D. A., Johnson, D. B. and Hodges, L. F., Testbed Evaluation of Virtual Environment Interaction Techniques., Teleoperators and Virtual Environments, 10(1), 75-95, 2001.

Bowman. D. A., Chen, J., Wingrave, C. A., Lucas, J., Ray, A., Polys, N. F., Li, Q., Haciahmetoglu, Y., Kim, J. S., Kim, S., Boehringe, S. and Ni, $\mathrm{T}$., New directions in $3 D$ user interfaces, The International Journal of Virtual Reality, 5(2), 3-14, 2006.

Bowman, D. A., Badillo, B. and Andmanek, D., "Evaluating the need for display-specific and device-specific 3D interaction techniques." In 
Proceedings of the Virtual Reality International Conference, 2007.

Dachselt, R. and Hübner., A. Three-dimensional menus: a survey and taxonomy., In Computers and Graphics, 31(1), 53-65, 2007.

Gerber, D. and Bechmann, D., "Design and evaluation of the ring menu in virtual environments.", In: IPT 2004: Eighth immersive projection technology workshop, Ames, IA, USA, 2004.

Gerber, D. and Bechmann, D., "The spin menu: A menu system for virtual environments." IEEE Conference on Virtual Reality, (pp 271-272), 2005.

Jacoby, R. H. and Ellis, S. R., "Using virtual menus in a virtual environment". In Course Notes 43 - Implementing Virtual Reality - pages 7.1 to 7.8. SIGGRAPH'93, 20th International Conference on Computer Graphics llild Interactive Techniques (Anallcim, August 1993). New York: ACM, 1993.

Kim, N., Kim, G. J., Park, C.-M., Lee, I. and Lim, S.H., Multimodal menu presentation and selection in immersive virtual environments. In: Proceedings of IEEE Virtual Reality, 2000.

Mine, M. (1995). Virtual Environment Interaction Techniques. UNC Chapel Hill Computer Science Technical Report TR95-018.

Vanacken, L., Grossman, T. and Coninx, K., Multimodal Selection Techniques for Dense and Occluded 3D Virtual Environments, International Journal of Human-Computer Studies, 67(3), 237-255, 2009.

Ware, C. and Franck, G., Evaluating stereo and motion cues for visualizing information nets in three dimensions. ACM Transactions on Graphics, 15(2), 121-140, 1996.

Ye, J., Badiyani, S., Raja, V. and Schlegel, T., Applications of Virtual Reality in Product Design Evaluation. Human-Computer Interaction part IV, HCII 2007, LNCS 4553, 1190-1199, 2007.

\section{Author listings}

Jaeho Kim: jeokim@korea.ac.kr

Highest degree: BS, Department of Industrial System and Information

Engineering, Korea University

Position title: MS. Candidate, Graduate School of Industrial Management

and Security, Korea University

Areas of interest: Product Development, Universal Design(UD)
Sungwook Jung: saver@korea.ac.kr

Highest degree: MS, Department of Industrial System and Information Engineering, Korea University

Position title: $\mathrm{PhD}$. Candidate, Graduate School of Industrial

Management and Security, Korea University

Areas of interest: Product Development, Affective Engineering

Jaeho Choe: jhchoe@daejin.ac.kr

Highest degree: PhD, Department of Industrial Engineering, POSTECH

Position title: Professor, Department of Industrial and system

Engineering, Daejin University

Areas of interest: Product Development and Design

Eui S. Jung: ejung@korea.ac.kr

Highest degree: $\mathrm{PhD}$, Department of Industrial Engineering, Pennsylvania State University

Position title: Professor, Department of Industrial and Management Engineering, Korea University

Areas of interest: Poduct Development, Affective Engineering,

Ergonomics

Date Received : 2011-05-18

Date Revised :2011-06-21

Date Accepted : 2011-06-21 